\title{
Molecular Analysis of Rates of Metal Reduction andMetabolic State of Geobacter Species During in situ Uranium Bioremediation
}

\author{
Lovley, Derek R. \\ University of Massachusetts
}

\begin{abstract}
RESULTS TO DATE: This report summarizes progress from June 2004 through April 2005. Research focused on monitoring the in situ rates of metabolism and the metabolic state of Geobacteraceae during in situ bioremediation of uranium at the field study site in Rifle, Colorado. As detailed below, it was demonstrated for the first time that it is possible to quantify in situ levels of transcripts for key metabolic genes and from this information infer not only rates of electron transfer to metals, but also nutrient limitations which might be limiting this process.
\end{abstract}

As summarized in last year's report, initial pure culture studies had suggested that expression of the novel citrate synthase in Geobacter sulfurreducens was related to rates of $\mathrm{Fe}(\mathrm{III})$ reduction. This was further confirmed in detailed studies in which G. sulfurreducens was grown in chemostats with Fe(III) as the electron acceptor, or in studies in which electrodes served as the electron acceptor. The phylogeny of the citrate synthase was studied not only in pure cultures, but also in various subsurface environments in which Geobacteraceae predominate. Primers designed from these studies were used to quantify levels of transcripts for citrate synthase in various subsurface environments, with special emphasis on the 2004 field experiment at the Rifle site. At the Rifle site, levels of Geobacteraceae citrate synthase increased as acetate entered the treatment zone. When acetate levels dropped when the acetate supply was temporarily disrupted, citrate synthase transcript levels dropped. As the acetate supply resumed and acetate concentrations increased, the levels of citrate synthase transcript levels again increased in direct proportion to acetate availability. These studies clearly demonstrated that the metabolism of the Geobacter species that are responsible for in situ uranium bioremediation is limited by the availability of acetate and that it is possible to monitor acetate metabolism, and hence electron transfer to metals, by quantifying in situ levels of transcripts for citrate synthase. A manuscript summarizing these studies has been submitted to Applied and Environmental Microbiology.

Field studies at the Rifle study site have demonstrated that as Fe(III) oxides become depleted near the acetate injection zone, the numbers of Geobacter species decline and $\mathrm{U}(\mathrm{VI})$ reduction becomes inhibited as sulfate reducers become predominant. Therefore, it is important to track the activity of sulfate reducers as well as Geobacter species. Studies with pure cultures and aquatic sediments in which sulfate reduction was the predominant terminal electron accepting process indicated that monitoring levels of transcripts for dissimilatory sulfite reductase, a highly conserved gene involved in sulfate reduction, provided an indication of rates of sulfate reduction. A manuscript summarizing these results has been completed and will be submitted to Applied and Environmental Microbiology. The next step will be to evaluate the expression of the dissimilatory sulfate reductase at the Rifle site.

Studies in our laboratory have recently identified the gene for a novel, multicopper-containing outer membrane protein, designated $\mathrm{OmpB}$, that is highly conserved among acetate-oxidizing Geobacteraceae and is specifically required for reduction of insoluble Fe(III) oxides, the electron acceptor supporting the growth of Geobacter species during in situ uranium bioremediation. Evaluation of the expression of the OmpB gene revealed that it is expressed constitutively, with similar transcript levels at different rates of $\mathrm{Fe}(\mathrm{III})$ reduction in chemostat cultures. Monitoring of the transcript levels for OmpB during in situ uranium bioremediation revealed that, in contrast to expression of citrate synthase, expression of the OmpB gene did not track with acetate availability, indicating that the OmpB gene is also constitutively expressed in situ. This is significant because it provides a Geobacter-specific gene that can be used to normalize the results of the expression of genes, such as citrate synthase, that are not expressed constitutively. A manuscript summarizing these results has been written in preparation for publication in Applied and Environmental Microbiology. 
It is also important to be able to track the metabolic status of microorganisms during bioremediation to determine if the availability of key nutrients might be limiting rates of bioremediation. As noted in last year?s report, initial studies had been conducted on the possibility of using expression of nifD, a gene involved in nitrogen fixation, to determine whether Geobacteraceae were limited by the availability of fixed nitrogen. A paper summarizing these studies was accepted and published in Applied and Environmental Microbiology. Studies on nifD were continued in 2004. Levels of nifD transcripts were quantified every other day during the 2004 field experiment at the Rifle site. The results clearly demonstrated that Geobacter species were limited for fixed nitrogen during bioremediation. This suggests that adding fixed nitrogen along with acetate might increase the growth and/or activity of Geobacteraceae in situ. A manuscript describing these results and the overall growth and activity of the Geobacteraceae during the 2004 field experiment in which community composition and activity were intensely monitored in an unprecedented, detailed manner is in preparation.

Results of the 2003 field experiment, detailed in last year?s progress report, were summarized in a manuscript which has now been accepted for publication in Applied and Environmental Microbiology.

The studies of in situ uranium bioremediation with Geobacter species continued to receive significant press attention. For details please visit www.geobacter.org.

DELIVERABLES: 1. Chin, K.-J., A. Esteve-Nunez, and D. R. Lovley. 2004. Correlation between mRNA levels for respiratory genes and rates of respiration in Geobacter sulfurreducens. Appl. Environ. Microbiol. 70:5183-5189. 2. Holmes, D. E., K. P. Nevin, and D. R. Lovley. 2004. Comparison of 16S rRNA, nifD, recA, gyrB, rpoB, and fusA genes within the family Geobacteraceae. Int. J. Syst. Evol. Microbiol. 54:15911599. 3. Holmes, D. E., K. P. Nevin, and D. R. Lovley. 2004. In situ expression of Geobacteraceae nifD in subsurface sediments. Appl. Environ. Microbiol. 70:7251-7259. 4. Holmes, D. E., K. P. Nevin, O. N. R.A., J. E. Ward, L. A. Adams, T. L. Woodward, and D. R. Lovley. 2005. Potential for quantifying expression of Geobacteraceae citrate synthase gene to assess the activity of Geobacteraceae in the subsurface and on current harvesting-electrodes. Appl. Environ. Microbiol.:(submitted). 5. Vironis, H. A., R. T. Anderson, I. Ortiz-Bernad, K. R. O'Neil, C. T. Resch, A. D. Peacock, R. Dayvault, D. C. White, P. E. Long, and D. R. Lovley. 2004. Microbiological and geochemical heterogeneity in an in situ uranium bioremediation field site. Appl. Environ. Microbiol. 71:(in press). 